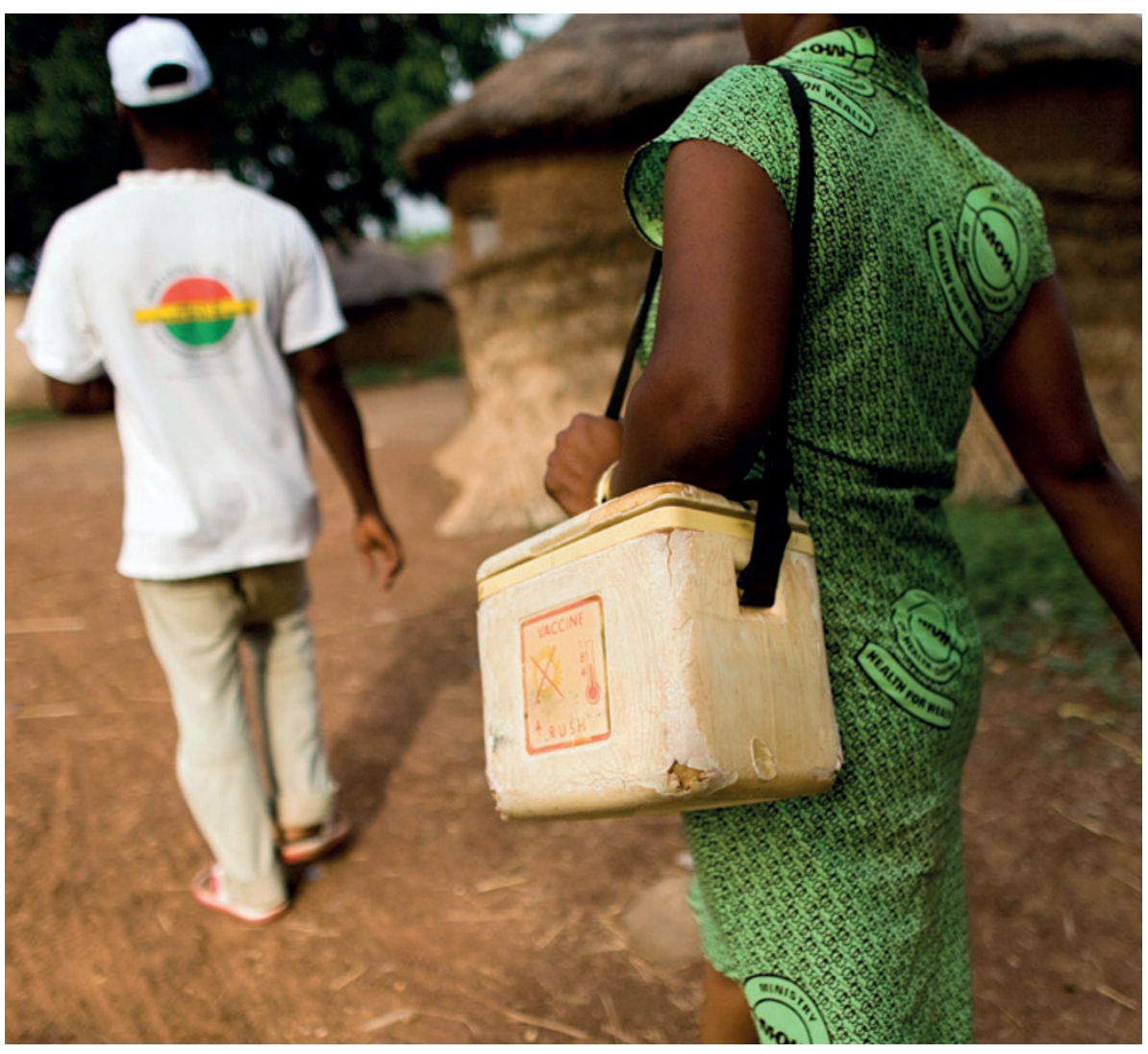

A village nurse carries her characteristic 'vaccine bag', an insulated case that is crucial for keeping vaccines at the right temperature, in Vailankanni near Tamil Nadu in India.

\title{
LOGISTICS
}

\section{Keeping cool}

\section{Extreme temperatures damage vaccines. Efforts are underway to find better ways to deliver the goods.}

\section{NEIL SAVAGE}

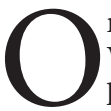
ne hot November afternoon in the West African country of Benin, a line of rural villagers takes shade beneath trees as they wait their turn for a shot. Vaccinators have travelled here, and to other villages in the Banikoara district, on bicycles, trucks and motorbikes bearing insulated containers holding a vaccine against a strain of meningococcal meningitis, which is endemic to this part of the world.

This vaccination campaign in 2012 was different from those that went before it: none of the vaccinators were toting the heavy ice packs used to keep vaccines between $2^{\circ} \mathrm{C}$ and $8^{\circ} \mathrm{C}$ as recommended by the World Health Organization (WHO). The vaccine, MenAfriVac, was approved for use even after being stored at up to $40^{\circ} \mathrm{C}$ for as long as four days.

The ability to transport doses of a vaccine outside the so-called cold chain
- the series of refrigerators, refrigerated trucks, and ice-chilled carriers that keep the medicine between $2^{\circ} \mathrm{C}$ and $8^{\circ} \mathrm{C}$ - will lower the cost of vaccination, improve the delivery of vaccines in the developing world, and give health-care workers greater flexibility in reaching those in need, says Simona Zipursky, a public health specialist in the WHO's Expanded Programme on Immunization. No longer would healthcare workers have to travel to the nearest city overnight just to freeze ice packs, and they could stay in the field longer without having to throw away vaccine that had expired from the heat. "Four days isn't four years, but it opens the pathway," Zipursky says.

Maintaining the cold chain is a major challenge to the goal of delivering vaccines to everyone who needs them, particularly in the developing world. So researchers are looking for new vaccine formulations and delivery systems that don't depend on such a narrow range of temperatures. At the same time, they're working on ways to improve the cold chain, with high-tech containers for trans porting vaccines and information technology to monitor cold storage.

\section{BREAKING THE CHAIN}

MenAfriVac was the first vaccine to be approved for use outside the cold chain. It was developed in a collaboration between the WHO and PATH, the Program for Appropriate Technology in Health, an international not-for-profit organization based in Seattle, Washington. Immunization specialists have long known that the standards for vaccine expiration were very conservative, Zipursky says. Vaccine makers generally run accelerated stability studies: stress tests in which they expose the medicine to high temperatures to see when it starts to break down. The collaboration added a few additional tests to measure various ways in which the chemistry of this vaccine could change to render it useless, and decided it would still be safe and effective after up to four days at $40^{\circ} \mathrm{C}$. "In reality," Zipursky says, "we probably have somewhere near a few weeks."

Initial results suggest that MenAfriVac has greatly reduced the incidence of meningitis. A study in Chad during the 2012 infection season found only 2.5 cases of this strain of meningitis per 100,000 people where the vaccine had been distributed, compared to 43.8 cases per 100,000 where it had not been distributed.

Different vaccines vary in their ability to tolerate high temperatures, Zipursky says. The collaboration is looking at the temperature tolerance of other vaccines, including those for cholera, hepatitis B and yellow fever. Running these studies can be costly: manufacturers don't necessarily have all the needed data on shelf life, and "it's not as fast a process as we would like," she says.

Heat isn't vaccines' only enemy. "Freeze damage may potentially be a greater issue than heat damage to vaccines," says Debra Kristensen, who leads PATH's vaccine technologies group. Many vaccines contain adjuvants: chemical compounds such as aluminium salts that stimulate a better immune response. If a vaccine freezes, the aluminium compounds clump together and become ineffective, rendering the vaccine less potent. A 2007 analysis by PATH of several studies found that between $14 \%$ and $35 \%$ of vaccine shipments were exposed to sub-zero temperatures at one point during shipping or storage. Analysis of a smaller group of six studies found that, in multiple rounds of shipping and storage, nearly all vaccine shipments examined were exposed to freezing temperatures at some time.

"Essentially, the more you look for freeze exposure, the more you'll find it," says Dipika Matthias, a public health specialist at PATH 


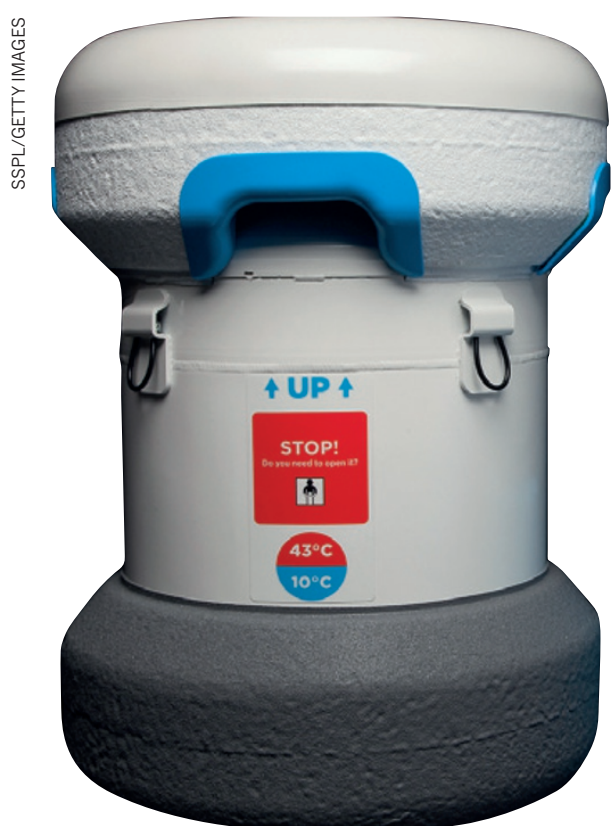

A state-of-the-art vaccine carrier keeps its cargo cool for longer.

and an author of the study. So researchers at PATH are experimenting with adding chemicals, such as glycerin, to act as an antifreeze in vaccines for polio, hepatitis B and diphtheria-pertussis-tetanus. The additives are considered nontoxic, but adding them to vaccines does require new rounds of safety testing to get regulatory approval. Because of the time and cost involved in such testing, Kristensen says it makes more sense to add stabilization agents to new vaccines under development rather than to existing ones. "It's an expensive process," she says."

Another approach is to move away from liquid vaccines entirely. Vaccine dried to a powder is more stable and doesn't require refrigeration. Here, vaccine makers can borrow technology that's been used in the food industry for decades, such as freeze-drying. This process freezes the vaccine quickly enough to minimize clumping of the adjuvants. The downside - in addition to the higher manufacturing cost - is that the dried vaccine needs to be mixed with liquid again before it can be injected. In areas with limited clean water and sterilization facilities, the reconstitution process could introduce contamination, Kristensen says.

A newer approach for vaccines, although still an old technology, is spray drying. The vaccine is passed through a nozzle and shot out as a fine spray of tiny droplets into a drying chamber filled with inert, heated gas. In addition to stabilizing the vaccine, the process also allows manufacturers to control the size of the particles, opening up new methods to deliver the vaccine. The rightsized particles can be easily absorbed through mucous membranes, so the vaccine could be inhaled instead of injected.
Diane Griffin, an immunologist at Johns Hopkins Bloomberg School of Public Health in Baltimore, Maryland, tested a powdered measles vaccine on a group of macaques. The powder was created with a spray-drying process developed at the University of Colorado, Boulder, by biotechnologist Robert Sievers. The powder was either applied on a mask strapped to the monkey's face or puffed into its nose with a squeezable bulb. The inoculation succeeded in protecting the monkeys against measles and caused no ill effects.

The powdered vaccine has since gone through a phase I clinical trial in India, where it was tested for safety on a group of already immunized adults. Griffin says the next stage is to test it in infants to see whether it promotes as effective an immune response as an injected vaccine. If it does, "it would make large scale immunization campaigns much easier," she says. "Not only are needles and syringes and the cold chain eliminated, but the vaccine comes in small, single-dose capsules that greatly reduce the volume for shipping and storage."

The same approach could be applied to vaccines for other diseases, such as rubella and mumps. Unfortunately, Griffin says, there are hurdles that have nothing to do with the science; researchers have been unable to secure funding to continue studying the powdered measles vaccine. "The vaccine community is very reluctant to do the testing and navigate the regulatory hurdles required to replace a cheap, successful vaccine with something even better," she says.

\section{STRONGER LINKS}

For the time being, vaccinators are relying on liquid vaccines. Researchers at Global Good, a collaboration between the research laboratory Intellectual Ventures, of Bellevue, Washington, and the Bill \& Melinda Gates Foundation, are developing a vaccine carrier designed to keep medicine cold while surviving journeys along bumpy roads in areas without electricity.

The carrier is essentially a high-tech version of a thermos: two stainless-steel cylinders with a vacuum in between for insulation. The cylinders are corrugated, increasing the surface area, so that heat from the outside has to follow a much longer path to reach inside the container. It's wrapped in thin, multiple layers of aluminium foil that act as an insulator and reflect heat from the container. "We have specifically designed it to fight the three ways that heat moves: convection, conduction, and radiation," says Geoff Deane, vice president at Intellectual Ventures. Only $1 \mathrm{~W}$ of heat energy leaks into the device, he says, compared to $30-50 \mathrm{~W}$ for the best insulating styrofoam carriers. Ice in separate plastic containers inside the carrier keeps it cool but doesn't come into direct contact with the vaccine, and keeps the vaccine fresh for over a month. Simple, battery-powered electronics record the temperature being maintained, while a GPS device tracks the carrier's location. All this information can be transmitted via SMS message. "Once a day it phones home and reports that data," Deane says. The team is performing field trials of the carrier in West Africa and a manufacturing partner is preparing to produce the device. Deane expects it will be ready for sale to vaccine distributors by mid- 2014 .

Such monitoring and tracking technology is already in use across the cold chain, says Liz Peloso, who heads PATH's Better Immunization Data Initiative, a new five-year, US $\$ 19.5$ million project funded by the Gates Foundation. For instance, many health centres have US\$20 electronic tags that monitor temperatures in storage refrigerators, and then send the data to a central repository. But these technologies are not currently used to their full potential, says Peloso.

In many cases, she says, workers at the local health centre are not privy to the information, which does not reach a central health district until weeks later. Or one health centre might be out of a vaccine while a neighbouring centre a few kilometres away has vials it can't use before they expire, but there's no way to share that information and, therefore, the extra vaccine. There needs to be a culture change that encourages local workers to identify and solve problems, Peloso says; if a tag records

"Maintaining
the cold chain
is a major
challenge to
the goal of
delivering
vaccines to
everyone who
needs them."

that a refrigerator temperature is going out of range at certain times, the local workers should know to check whether there's a problem with the power supply or someone is leaving the door open. Such simple responses usually aren't happening, she says. The initiative's aim is to develop protocols that take advantage of collected data to answer the question: "How do you turn the data points into information that somebody can use?"

The Better Immunization Data Initiative will launch its first project in Tanzania later in 2014, then start adding other countries where the infrastructure - electricity and communications - is stable enough to take advantage of the technology.

Ultimately, protocols designed to improve immunization could also spill into other areas, such as perinatal care, AIDS treatment and nutrition. "If you can make these things more efficient and effective," Peloso says, "you can really make a difference in how a country's health system operates."

Neil Savage is a science and technology writer based in Lowell, Massachusetts. 